\title{
Re-establishment of VWF-dependent Weibel-Palade bodies in VWD endothelial cells
}

\author{
Sandra L. Haberichter, Elizabeth P. Merricks, Scot A. Fahs, Pamela A. Christopherson, \\ Timothy C. Nichols, and Robert R. Montgomery \\ Department of Pediatrics, Medical College of Wisconsin, Milwaukee; the Blood Research \\ Institute, Blood Center of Southeastern Wisconsin, Milwaukee; the Children's Research Institute; \\ Children's Hospital of Wisconsin, Milwaukee; and the Department of Pathology and Laboratory \\ Medicine, University of North Carolina School of Medicine, Chapel Hill.
}

\begin{abstract}
Type 3 von Willebrand disease (VWD) is a severe hemorrhagic defect in humans. We now identify the homozygous mutation in the Chapel Hill strain of canine type 3 VWD that results in premature termination of von Willebrand factor (VWF) protein synthesis. We cultured endothelium from VWD and normal dogs to study intracellular VWF trafficking and WeibelPalade body formation. Weibel-Palade bodies could not be identified in the canine VWD aortic endothelial cells (VWD-AECs) by P-selectin, VWFpp, or VWF immunostaining and confocal microscopy. We demonstrate the reestablishment of Weibel-Palade bodies that recruit endogenous P-selectin by expressing wild-type VWF in VWD-AECs. Expression of mutant VWF proteins confirmed that VWF multimerization is not necessary for Weibel-Palade body creation. Although the VWF propeptide is required for the formation of Weibel-Palade bodies, it cannot independently induce the formation of the granule. These VWF-null endothelial cells provide a unique opportunity to examine the biogenesis of Weibel-Palade bodies in endothelium from a canine model of type 3 VWD.
\end{abstract}

\section{Introduction}

von Willebrand factor (VWF) is a large, multimeric glycoprotein that mediates platelet adhesion to subendothelial tissue at the site of vascular injury. VWF is also the carrier protein for coagulation factor VIII (FVIII). ${ }^{1,2}$ Defects in VWF function or decreased levels of VWF cause von Willebrand disease (VWD). ${ }^{3,4} \mathrm{VWF}$ is synthe sized as pre-pro-VWF consisting of a 22-amino acid (aa) signal peptide, 741-aa propeptide, and 2050-aa mature VWF monomer. ${ }^{5-9}$ VWF undergoes extensive intracellular modification. ${ }^{10}$ The pro-VWF forms carboxy-terminal dimers in the endoplasmic reticulum where signal peptide is removed. The VWF molecule is glycosylated, carbohydrate processed, and sulfated. ${ }^{11,12}$ In the Golgi, VWF dimers form amino-terminal multimers, and propeptide is cleaved, presumably by the enzyme furin. ${ }^{13}$ VWF is stored together with its propeptide in regulated secretory vesicles, Weibel-Palade bodies in endothelial cells and a-granules in megakaryocytes or platelets. ${ }^{6}$

The VWF released from Weibel-Palade bodies consists of very high-molecular-weight VWF multimers that are the most active for binding to subendothelial tissue and in platelet-platelet interactions. ${ }^{14-16}$ Thus, the multimerization and regulated storage of VWF are important for

Copyright 2011 by The American Society of Hematology; all rights reserved.@ 2005 by The American Society of Hematology Reprints: Sandra L. Haberichter, Department of Pediatrics, Medical College of Wisconsin, 8701 Watertown Plank Rd, Milwaukee, WI 53226; shaberic@mail.mcw.edu.. 
initiation of clot formation following injury to a vessel. The large propeptide, VWFpp, is required for multimerization and regulated storage of VWF. ${ }^{17,18}$ Previous studies in our laboratory have demonstrated that VWF is sorted to regulated storage vesicles in a VWFppdependent manner. ${ }^{19,20}$ Our studies were carried out using the mouse pituitary model cell line AtT-20 and bovine aortic endothelial cells (BAECs) that have endogenous-regulated storage granules containing adrenocorticotrophic hormone (ACTH) or bovine VWF, respectively. We have demonstrated that the propeptide independently sorts to endogenous storage granules in these cells and co-traffics mature VWF multimers through a noncovalent association. ${ }^{19,21}$ However, because of the presence of endogenous-regulated secretory vesicles, these studies could not address the biogenesis of the regulated storage vesicle.

Although the biogenesis and exocytosis of Weibel-Palade bodies has been the subject of investigation, the mechanisms are not well defined. ${ }^{14,22,23}$ To pursue our studies on intracellular VWF trafficking and Weibel-Palade body biogenesis, we cultured endothelium from the Chapel Hill strain of canine (K9) type 3 VWD. Type 3 VWD is a severe bleeding disorder in humans, characterized by a complete absence of VWF. ${ }^{3}$ Natural animal models of type 3 VWD exist in pigs and dogs, as well as a VWF-null transgenic mouse model. ${ }^{24,25}$ The type 3 VWD dogs have been maintained in Chapel Hill for many years, but the molecular defect causing the disease had not been identified. ${ }^{26}$ Here we first identify the mutation in these dogs that results in premature termination of VWF protein synthesis. Endothelial cells were obtained from the aortas of VWD dogs, and the absence of WeibelPalade bodies, VWFpp, and VWF were confirmed. We have examined the ability of expressed wild-type and mutant VWF, or VWFpp, to generate storage granules that recruit endogenous P-selectin to reestablish Weibel-Palade bodies in the VWD endothelial cells. The availability of a VWF-null, nontransformed endothelial cell has thus provided a beneficial model for investigating the mechanisms involved in Weibel-Palade body formation.

\section{Materials and methods}

\section{Antibodies}

Anti-VWF antibodies 280.1, AVW-1, AVW-5, and 105.4 and the polyclonal, Edwina, were produced by our laboratory. A rabbit polyclonal anti-human VWF was purchased from Dako (Carpinteria, CA). The monoclonals AVW-5 and 105.4 and the polyclonal antibodies recognize both human and canine VWF. The antibody 280.1 is specific for canine VWF and AVW-1 is human VWF specific. Anti-VWFpp monoclonal antibodies 239.1 to 239.11, $242.4,242.5,275.1$, and 275.2 were also produced by our laboratory; 239.1, 239.7, 239.8, $239.11,242.4$, and 242.5 recognize canine VWFpp in addition to human, while the 275.1 and 275.2 only recognize canine propeptide. The anti-human platelet endothelial cell adhesion molecule (PECAM) antibody, PECAM 1.3, was also produced in our laboratory. Other antibodies used include rabbit polyclonal anti-P-selectin (BD Bioscience, San Jose, CA), and goat-anti-C3 (Sigma Chemical, St. Louis, MO). Nonimmune mouse and rabbit immunoglobulin $\mathrm{G}$ (IgG) were purchased from Jackson Immunoresearch (West Grove, PA) and used as isotype controls. Secondary antibodies used for immunofluorescence detection included goat anti-rabbit and anti-mouse $\left.\operatorname{IgG}(\mathrm{H}+\mathrm{L})\left[\mathrm{F}(\mathrm{Ab})_{2}\right)_{2}\right]$ fragments conjugated with either AlexaFluor-488 or AlexaFluor-568 (Molecular Probes, Eugene, OR), or donkey antirabbit, anti-goat, or anti-mouse $\operatorname{IgG}(\mathrm{H}+\mathrm{L})\left[\mathrm{F}\left(\mathrm{Ab}^{\prime}\right)_{2}\right]$ fragments (Jackson Immunoresearch) conjugated with Texas Red (TXR), Red-X, or fluorescein isothiocyanate (FITC).

\section{Constructs}

The human and canine VWF, VWF propeptide (VWFpp), and propeptide-deleted VWF ( $\Delta$ pro) expression vectors were constructed as previously described..$^{21}$ Briefly, VWFpp 
expression vectors contain signal peptide and VWFpp sequence followed by a stop codon with the VWF sequence deleted. The $\Delta$ pro expression vectors contain signal peptide followed directly by mature VWF sequence. The Y87S-VWF is a full-length VWF construct that encodes a point mutation in the D1 domain of the VWF propeptide as previously described. ${ }^{27}$ VWFpp*C3a vector expresses VWF signal peptide and VWFpp with a disrupted furin cleavage site followed directly by the a-chain of human complement $\mathrm{C} 3$ as previously described. ${ }^{19}$

\section{Sequencing}

White blood cell DNA was purified from normal and VWD dogs from the colony maintained at the University of North Carolina, Chapel Hill. In particular, a segment of genomic sequence spanning from base 228 in exon 4 to base 532 in exon 5 of the $V W F$ gene was amplified by polymerase chain reaction (PCR) using sense primer K9-VWF s228 to 250 (5'-C CAA AAT GGC AAA AGA GTG AGC C-3') and antisense primer K9-VWF a532 to 510 (5'-C TTC TTG AGT CCT GAA GTC ATC C-3'). Directly sequencing the PCR product from 2 VWD dogs using the same antisense primer yielded a single sequence with a nucleotide deletion of the cytosine at base $255(\Delta \mathrm{C} 255)$ of normal canine VWF cDNA. The PCR products were cloned and sequenced in both directions to confirm the mutation.

\section{Cell culture and transfections}

Endothelial cells were harvested by digestion with $1 \mathrm{mg} / \mathrm{mL}$ collagenase from the aortas obtained from normal and VWD dogs. The harvested canine aortic endothelial cells (AECs) were cultured in Medium 199 (Mediatech, Holy Hill, FL) supplemented with 10\% fetal bovine serum (FBS) and grown on tissue culture dishes coated with $2 \%$ gelatin, at $37^{\circ} \mathrm{C}$ in an atmosphere of $5 \% \mathrm{CO}_{2}$. Cells were transiently transfected by Nucleofection (Amaxa, Cologne, Germany) according to manufacturer's instructions. Negative controls were either nontransfected or mock transfected with expression vector lacking the insert. When transfected cells reached confluency (3-7 days after nucleofection), conditioned media were harvested from the cells, centrifuged to remove debris, and frozen at $-80^{\circ} \mathrm{C}$ for further analysis. Washed cells were either fixed for intracellular staining or used for secretion studies. To assess stimulated release of expressed VWF proteins, cells were washed with Hanks balanced salt solution (HBSS), followed by OptiMEM washes, and then incubated for 30 minutes with either $2 \mu \mathrm{M}$ phorbol myristate acetate (PMA) or OptiMEM (control). Releasates were harvested, and secreted VWF protein was quantitated by either VWFpp or VWF enzyme-linked immunosorbent assay (ELISA).

\section{Confocal immunofluorescence microscopy}

Cells were analyzed for the intracellular location of VWF, VWFpp, and other proteins by immunofluorescence using antibody staining and confocal laser scanning microscopy in the Imaging Core of the Medical College of Wisconsin using a Leica (Heidelberg, Germany) TCS SP 2 confocal laser imaging system and software with $63 \times$ or $100 \times$ objective lenses as previously described. ${ }^{20}$ Cells were grown on 2 -well chamber slides coated with $0.2 \%$ gelatin and cross-linked with $0.5 \%$ glutaraldehyde. Confluent cells were fixed using $3.7 \%$ (vol/vol) buffered formalin, permeabilized in $1 \%$ Triton X-100, and blocked with $2 \%$ normal goat serum or donkey serum in HBSS. Cells were incubated at $4^{\circ} \mathrm{C}$ overnight in primary antibodies diluted in HBSS containing $1 \%$ bovine serum albumin (BSA). The concentration of primary antibody varied from 2 to $5 \mu \mathrm{g} / \mathrm{mL}$. Cells were then washed 3 times with HBSS. All immunostaining experiments used normal mouse and rabbit IgG for isotype controls. Cells were incubated in secondary antibodies diluted 1:1000 in HBSS containing 1\% BSA for 30 minutes at room temperature. Cells were then washed 3 times with HBSS and mounted with cover glass using Vectashield (Vector Labs, Burlingame, CA). 


\section{Multimer analysis}

VWF in the conditioned medium of transfected endothelial cells was analyzed by electrophoresis through a $0.8 \%(\mathrm{wt} / \mathrm{vol}) \mathrm{HGT}(\mathrm{P})$ agarose (FMC Bioproducts, Rockland, ME) stacking gel and $2 \%(\mathrm{wt} / \mathrm{vol}) \mathrm{HGT}(\mathrm{P})$ agarose running gel containing $0.1 \%$ sodium dodecyl sulfate (SDS) for 16 hours at $40 \mathrm{~V}$ using the Laemmli buffer system with transfer and Western blotting as previously described. ${ }^{21}$ Submarine-agarose gel electrophoresis was performed as previously described. ${ }^{27}$

\section{Results}

The VWD dog colony has been maintained in Chapel Hill for many years. Although the bleeding disorder in these dogs has been established as type $3 \mathrm{VWD}$, the molecular defect causing the disease had not been determined. ${ }^{26,28}$ Since our laboratory had previously identified the sequence of normal canine VWF, we carried out sequencing of the $V W F$ gene using genomic DNA obtained from the VWD dog. ${ }^{21}$ In particular, a segment of genomic sequence spanning from exon 4 to exon 5 of the $V W F$ gene was amplified by PCR. The 591-base pair (bp) PCR product included bases 228 to 323 in exon 4, the 286-bp intron 4, and bases 324 to 532 in exon 5. A comparison of the normal and VWD sequence is shown in Figure 1. Sequencing revealed a single (homozygous) nucleotide deletion $(\Delta \mathrm{C} 255)$ in exon 4 of the VWF propeptide (VWFpp), resulting in a frameshift mutation at amino acid 85 of the VWF protein. After 34 altered amino acids, the shifted reading frame results in a TAG stop codon at base 359 , leading to premature termination of VWF protein synthesis. This mutation accounts for the complete absence of VWF in the canine VWD plasma.

Although no VWF is detected in the VWD dog plasma, we questioned whether any VWF or VWFpp protein could be detected in the endothelial cells of the VWD dogs. If these endothelial cells were indeed VWF-null they would provide a valuable tool for studying the intracellular trafficking of VWF. VWF trafficking and biogenesis of Weibel-Palade bodies have previously been studied by our laboratory and others using transformed model cell lines. ${ }^{17,20,21,29-31}$ A VWF-null, nontransformed endothelial cell has not been available. We isolated and characterized the AECs from normal dogs to compare with those obtained from the VWD dogs. Harvested cells were grown on gelatin, immunostained as described in "Materials and methods," and examined by confocal microscopy. Immunostaining for the endothelial cell marker PECAM-1 (CD31) as shown in Figure 2A demonstrated that a homogeneous population of normal AECs had been cultured. Immunostaining with a VWF polyclonal antibody confirmed that the normal canine endothelial cells also express VWF (Figure 2B). Immunostaining for VWFpp and VWF demonstrated colocalization of VWF with VWFpp in granules (Figure 2D-F), similar to the Weibel-Palade body distribution in human umbilical vein endothelial cells (HUVECs). ${ }^{6}$ Weibel-Palade bodies contain the membrane protein P-selectin. ${ }^{32,33}$ Dual-label immunostaining of canine AECs revealed that endogenous P-selectin was present in the granules containing VWF (Figure 2G-I). These data confirm that normal canine AECs contain characteristic Weibel-Palade bodies and are similar to human endothelial cells.

We next harvested and cultured endothelial cells from the VWD dogs. Immunostaining for PECAM-1 demonstrated a homogeneous population of VWD-AECs has been cultured (Figure 2J). We could not identify a significant difference in morphology between normal and VWD endothelial cells. We also stained for VWF, using the polyclonal antibody that detected the canine VWF in the normal endothelial cells, and no VWF was detected (Figure $2 \mathrm{~K}, \mathrm{~N}$ ). No VWF was detected using monoclonal antibodies specific for canine VWF (data not shown). Although sequencing data predicted the potential synthesis of the first 85 amino acids of propeptide, we could not detect VWF mRNA by reverse transcriptase (RT)-PCR using canine specific probes (data not shown). The canine VWD-AECs were immunostained 
by using a mix of several monoclonal antibodies that recognize canine VWFpp, and no VWFpp expression was detected (Figure 2M). A monoclonal antibody specific for canine VWFpp also failed to detect VWFpp expression (Figure 2Q). These findings demonstrate that the AECs obtained from VWD dogs are VWF and VWFpp null at both a protein and an mRNA level.

Having established the absence of both VWFpp and VWF in the canine VWD-AECs, we examined the intracellular localization of P-selectin, a recognized marker of the WeibelPalade body. The staining of P-selectin was faint and fairly diffuse. Although several very small granules were observed, most likely these were endosomes/lysosomes (Figure 2P). This would be consistent with previous findings that in the absence of VWF, in cells lacking a regulated secretory pathway, $\mathrm{P}$-selectin is delivered to the lysosome and degraded. ${ }^{34}$ No apparent Weibel-Palade bodies are detected in the canine VWD-AECs.

The VWF-null canine AECs provide a unique opportunity to study Weibel-Palade body biogenesis in a naturally occurring animal model of type 3 VWD. We tested the hypothesis that formation of Weibel-Palade bodies would be induced by expression of either wild-type human or canine VWF. Full-length VWF constructs were introduced by nucleofection of the canine VWD-AECs. Transfected cells were immunostained for VWFpp and VWF and examined by confocal microscopy. In cells expressing canine VWF (data not shown) or human VWF (Figure 3A-C), both VWFpp and VWF appeared to be colocalized in granules. In model cell lines, the recruitment of P-selectin to VWF-containing granules has been used to authenticate the formation of Weibel-Palade bodies. ${ }^{30,31}$ To confirm the formation of Weibel-Palade bodies we examined whether VWF-containing granules in nucleofected canine VWD-AECs would recruit endogenous P-selectin. In cells expressing full-length human VWF (data not shown) or canine VWF (Figure 3D-F), endogenous P-selectin colocalized with the VWF in granules, similar to that in the normal canine endothelial cell. These data indicate that the canine VWD-AECs will support the formation of Weibel-Palade bodies when VWF is expressed and the Weibel-Palade body distribution of P-selectin is reestablished.

Previous work in our laboratory demonstrated that the granular storage of VWF in the AtT-20 model cell line was not dependent on multimerization of VWF. ${ }^{21}$ To confirm this in an endothelial cell, we expressed 2 dimeric VWF proteins. The first protein is propeptidedeleted VWF ( $\Delta$ pro) that contains signal peptide followed directly by the mature VWF sequence. When canine $\Delta$ pro (not shown) or human $\Delta$ pro (Figure 3G-I) were expressed in the canine VWD-AECs, only diffuse VWF staining was observed, no apparent VWFcontaining granules could be detected. The P-selectin distribution remained diffuse with the presence of some small granules (Figure 3H) and did not colocalize with VWF (Figure 3I). The second dimeric VWF protein we expressed is a full-length, propeptide-containing mutant with a point mutation in the D1 domain of the propeptide (Y87S) that our laboratory had previously characterized. ${ }^{27}$ Canine VWD-AECs expressing the Y87S-VWF demonstrated the presence of VWFpp and VWF colocalized in granules (Figure 3J-L), with some diffuse staining consistent with localization in the endoplasmic reticulum. Additionally, P-selectin was recruited to the granules in those cells that were expressing Y87S-VWF (not shown) verifying that multimerization is not required for granule formation.

To confirm that the propeptide is required for granule formation, we coexpressed $\Delta$ pro together with VWFpp as 2 separate constructs (in trans). We observed that nucleofected cells received and expressed both $\Delta$ pro and VWFpp rather than one or the other plasmid (not shown). Cells expressing both human VWFpp and $\Delta$ pro proteins displayed the presence of VWF (and presumably VWFpp) in granules that recruited endogenous P-selectin (Figure 
3M-O). These data indicate that only when $\Delta$ pro is coexpressed with VWFpp, in either cis or trans, can granules be formed that recruit endogenous P-selectin. Together, these data confirm earlier findings that propeptide is necessary for granule formation, $\Delta$ pro alone is not sufficient for formation of a Weibel-Palade body, and multimerization of the VWF is not required for granule biogenesis.

To assess the multimerization of expressed VWF proteins, we analyzed the culture supernatants of transfected cells by SDS-agarose electrophoresis and Western blotting (Figure 4A). No VWF multimers were detected in the supernatants of mock-transfected (lane 7) canine VWD-AECs. Both the wild-type canine and human full-length VWF were normally multimerized (lanes 2, 3). The lack of VWF multimerization of the Y87S-VWF and $\Delta$ pro proteins was confirmed (lanes 4,5 ). The conditioned media were also analyzed on submarine MetaPhor-agarose gels that better define lower molecular weight VWF proteins (Figure 4B). Both Y87S-VWF and $\Delta$ pro proteins formed only a dimeric VWF species (lanes $4,5)$. Examination of full-length human and canine VWF samples revealed that a higher molecular weight species (tetramer) migrated into the gel in addition to the VWF dimer (lanes 1,3). Densitometry revealed a similar percentage of VWF dimer and tetramer in human and canine samples (lanes 1,3; peaks 1,2). Expression of wild-type VWF proteins in canine VWD-AECs results in normal multimerization and secretion of VWF.

The propeptide is clearly necessary for formation of a VWF-containing Weibel-Palade body. Our previous studies in AtT-20 cells demonstrated that VWFpp independently traffics to ACTH-containing regulated secretory granules, but these studies could not address whether VWFpp could generate the granule. We exploited the absence of endogenous secretory granules in the canine VWD-AECs to examine whether the VWF propeptide is sufficient for Weibel-Palade body formation. Immunostaining and confocal microscopy of cells expressing either human VWFpp (Figure 5A-C) or canine VWFpp (Figure 5D-F) demonstrated mostly diffuse staining, although a number of small, VWFpp-containing granules were occasionally observed. We also examined the distribution of P-selectin in the VWFpp-expressing canine VWD-AECs. When either human VWFpp (Figure 5G-I) or canine VWFpp (data not shown) were expressed, the distribution of P-selectin was not altered. VWFpp and P-selectin did not appear to be colocalized, indicating that VWFpp alone is not sufficient for formation of a Weibel-Palade body that recruits endogenous Pselectin. These data suggest that in addition to VWFpp, the mature portion is necessary for the biogenesis of a P-selectin containing Weibel-Palade body.

We hypothesized that the mature portion of the VWF molecule was providing the dense core of protein needed for condensation, aggregation, and granule formation. We sought to determine whether another protein could provide the necessary aggregation. We reported a VWFpp fusion protein, VWFpp*C3a, that contains signal peptide and VWFpp with a disrupted furin cleavage site followed by the a-chain of complement C3. ${ }^{19}$ This VWFpp*C3a fusion protein is stored in granules in the AtT-20 cell line and sorts to endogenous, VWF-containing granules in bovine aortic endothelial cells. Due to the presence of endogenous secretory vesicles in these cells we could not address the biogenesis of secretory vesicles. Dual-immunostaining for VWFpp and C3a of VWD-AEC expressing VWFpp*C3a (Figure 5J-L) occasionally showed the presence of a few VWFpp and C3acontaining granules, although the distribution of VWFpp and C3a in most cells was diffuse. VWFpp*C3a-transfected cells were also dual labeled for P-selectin and VWFpp. Some transfected cells demonstrated the presence of VWFpp-containing granules; however, the VWFpp did not appear to colocalize with endogenous P-selectin (Figure 5M-O). While the C3a fused to VWFpp may be sufficient for aggregation and granule formation, these granules do not recruit P-selectin, indicating that the mature portion of VWF may be necessary for the recruitment of P-selectin to the Weibel-Palade body. 
To determine whether the granules formed by expression of VWF proteins are regulated secretory granules, we examined whether release of the expressed proteins could be induced by exposure to the secretagogue, PMA. Confluent transfected cells were exposed to either PMA or control for 45 minutes at $37^{\circ} \mathrm{C}$. The incubation media was collected, and the amount of VWF (Figure 6A) or VWFpp (Figure 6B) was determined by ELISA. We observed a significant increase in VWF secretion in response to PMA (429\% of control). The release of Y87S-VWF was also significantly increased (426\% of control). Expressed $\Delta$ pro did not appear to be released in response to PMA (123\% of control). The VWF expression levels of cells exposed to control were equivalent (Figure 6A). Additionally, the VWF expression levels over the 24-hour period prior to agonist stimulation were found to be similar for wild-type VWF, Y87S-VWF, and $\Delta$ pro, ranging from 3 to $6 \mathrm{mU} / \mathrm{mL}$. The VWFpp concentration for wild-type VWF and Y87S-VWF samples were also in this range, indicating a VWFpp/VWF ratio of approximately 1:1. In contrast to full-length VWF, we did not observe a significant increase in secretion of VWFpp (99\% of control) or VWFpp*C3a (100\% of control) in response to PMA (Figure 6B). The prestimulation expression level of VWFpp*C3a was decreased when compared with VWFpp, $5 \mathrm{mU} / \mathrm{mL}$ and $13 \mathrm{mU} / \mathrm{mL}$, respectively. Cells expressing both VWFpp and $\Delta$ pro were responsive to PMA stimulation, and a significant increase in VWFpp secretion (and presumably VWF) was observed (Figure 6, 729\% of control). In this case, the prestimulation expression levels of VWFpp and $\Delta$ pro were not proportional. VWFpp concentration was almost 3 times greater than $\Delta$ pro, $10 \mathrm{mU} / \mathrm{mL}$ and $3 \mathrm{mU} / \mathrm{mL}$, respectively. This disproportionate expression could account for the seemingly increased agonist-induced release of VWFpp when it is coexpressed with $\Delta$ pro. Together, these data indicate that full-length VWF, Y87S-VWF, and the trans expression of VWFpp and $\Delta$ pro result in formation of Weibel-Palade bodies that undergo agonist-induced exocytosis, while $\Delta$ pro, VWFpp, or VWFpp*C $3 a$ are not trafficked to the regulated storage pathway.

\section{Discussion}

In this study, we have identified the molecular defect causing the type 3 VWD in the dogs from the closed colony maintained in Chapel Hill. The $\Delta \mathrm{C} 255$ deletion in exon 4 in the codon corresponding to serine 85 of the VWF propeptide results in 34 amino acids of altered protein sequence and premature termination of VWF synthesis. This mutation accounts for the absence of VWF protein in the plasma of the VWD dogs. Interestingly, this is the same mutation that was identified in Scottish Terriers with type 3 VWD. ${ }^{35}$ Although the sequence predicts the synthesis of the first 85 amino acids of VWFpp followed by 34 altered amino acids, we could not detect any truncated VWFpp protein, nor did we detect VWF mRNA in the VWD aortic endothelial cells by RT-PCR using canine VWFpp specific primers. Together these data indicate that the VWD dogs do not synthesize any VWF.

We have confirmed the absence of VWFpp and VWF in the AECs obtained from the VWD dogs. Examination of the distribution of P-selectin in the VWF-null aortic endothelial cells verified the absence of recognizable Weibel-Palade bodies in contrast with normal canine AECs. The canine P-selectin appeared to be degraded rapidly as indicated by the faint, diffuse staining and localization in small granules presumed to be lysosomes, similar to the P-selectin distribution observed in the VWF-deficient knockout mouse. ${ }^{25,36} \mathrm{~A}$ similar degradation of P-selectin was demonstrated in the T24 human epithelial cell line that does not synthesize VWF. ${ }^{29}$ We were unable to definitively identify the P-selectin-containing granules as lysosomes due to lack of availability of antibodies cross-reacting with canine lysosomal proteins. However, the degradation of P-selectin in the absence of a regulated secretory pathway is well-defined as previously described. ${ }^{34,37,38}$ 
Many prior studies on VWF trafficking have been performed in transformed, nonendothelial cell lines. Several laboratories have demonstrated the colocalization of P-selectin with VWF in storage granules when VWF is coexpressed with P-selectin in non-endothelial cell lines. ${ }^{30,31}$ A VWF-null, nontransformed endothelial cell has not been available for exploring the intracellular processing and trafficking of VWF in its natural site of synthesis. We have developed a system to examine the formation of storage granules in a true endothelial cell that does not contain preexisting secretory vesicles, synthesizes P-selectin, and is responsive to secretagogue. Our results using the VWF-null endothelial cells corroborate many earlier findings. Our laboratory and other investigators have shown that multimerization and storage of VWF are unlinked, independent processes. ${ }^{17,21,27,30}$ The formation of Weibel-Palade bodies that recruit endogenous P-selectin when dimeric Y87SVWF is expressed in the canine VWD-AECs provides further evidence. The trans expression and granular storage of VWFpp and $\Delta$ pro has been demonstrated in both AtT-20 and CV-1 cell lines. ${ }^{39}$ In the canine VWD cells, the coexpression of VWFpp and $\Delta$ pro of either canine or human origin created Weibel-Palade bodies that recruited endogenous Pselectin and were released in response to PMA.

The question that has not been addressed in any prior studies is whether the VWFpp alone is sufficient for the formation of a Weibel-Palade body. Due to the presence of endogenousregulated secretory vesicles in AtT-20 and bovine aortic endothelial cells, granule biogenesis could not be addressed. While the propeptide clearly sorts independently to these endogenous secretory granules, the ability to generate the storage granule is a different question. Here we demonstrate that VWFpp alone does not appear to generate a bona fide Weibel-Palade body. Canine VWD-AECs expressing VWFpp showed mostly diffuse staining consistent with its localization in the endoplasmic reticulum. Although many small granules were occasionally observed, the nature of these granules is not clear. The lack of Pselectin recruitment, diminished size, and lack of agonist-induced release of the granules formed by VWFpp in the VWF-null endothelial cells indicate that Weibel-Palade bodies are not formed. While VWFpp is clearly required in addition to mature VWF ( $\Delta$ pro), VWFpp independently is not sufficient for Weibel-Palade body biogenesis.

Two hypotheses have been proposed that would explain the formation of storage vesicles. ${ }^{40,41}$ One hypothesis focuses on the selective aggregation or condensation of proteins in the trans-Golgi to trigger granule formation. The second proposes that regulated secretory proteins contain targeting signals that interact with sorting "receptors" that escort the proteins into the storage pathway. We considered whether the "mature" portion ( $\Delta$ pro) of VWF is providing the aggregation needed for protein condensation and granule budding, or whether an alternative protein could substitute for $\Delta \mathrm{pro}$. In a previous study we fused the achain of complement C3 (C3a) to VWFpp to demonstrate the ability of VWFpp to traffic an unrelated, non-secretory-granule protein to the regulated storage pathway, but could not address the biogenesis of the granule. ${ }^{19}$ In the VWF-null canine endothelial cells, we observed a subset of cells that contained distinct granules, in addition to a significant number of cells with diffuse staining consistent with endoplasmic reticulum localization of VWFpp*C3a. In the cells that showed granule formation, canine P-selectin did not appear to colocalize with the VWFpp*C3a-containing granules. The lack of P-selectin recruitment can be rationalized by 1 of 2 explanations. The first explanation is that the VWFpp*C3acontaining granules are not regulated secretory vesicles. We did not observe any agonistinduced release of VWFpp*C3a. However, only a small percentage of cells expressing VWFpp*C3a contained granules, and the amount of released protein may not be within limits of detection. An alternative explanation for the lack of P-selectin recruitment is that Pselectin may be brought to the Weibel-Palade body in a VWF-dependent manner. Prior studies support this hypothesis as P-selectin can be diverted from ACTH-containing granules in AtT-20 cells (when expressed in the absence of VWF) to granules induced by 
VWF expression that do not contain ACTH. ${ }^{31}$ The mechanism for this VWF-dependent recruitment of P-selectin to the Weibel-Palade body remains to be clarified.

Although VWFpp alone does not appear to induce the formation of a Weibel-Palade body, it is clearly indispensable in the processing and intracellular trafficking of VWF. Evidently, both VWFpp and mature VWF protein are required for creation of the regulated secretory vesicle to which P-selectin ultimately traffics. Whether VWFpp provides the "sorting signal" and mature VWF provides the core of aggregation remains unresolved. With the exception of type $3 \mathrm{VWD}$, there have been no reports of patients that lack Weibel-Palade bodies. In the VWF-deficient mouse, the loss of Weibel-Palade body localized P-selectin resulted in defective regulated secretion and loss of leukocyte recruitment. Recently, Pselectin has been shown to interact with endothelial-released ultra-large VWF (ULVWF) multimers. ${ }^{42} \mathrm{P}$-selectin may serve to tether the ULVWF to endothelial cells. In addition to the impact on P-selectin, the loss of Weibel-Palade bodies due to defective VWF would have an impact on several other proteins, including tissue-type plasminogen activator (t-PA), interleukin 8 (IL-8), and potentially factor VIII that can be stored with VWF. ${ }^{43-47}$ Thus, defining the complex mechanism of Weibel-Palade body biogenesis has significant biologic implications.

\section{Acknowledgments}

Supported by Hemophilia Association of New York (S.L.H.), Children's Hospital Research Foundation (S.L.H.), National Institutes of Health grants (HL-44612, HL-33721, HL-56027) (R.R.M.) and (HL-63 098) (T.C.N.).

\section{References}

1. Sadler JE. Biochemistry and genetics of von Willebrand factor. Annu Rev Biochem. 1998; 67:395424. [PubMed: 9759493]

2. Weiss HJ, Sussman II, Hoyer LW. Stabilization of factor VIII in plasma by the von Willebrand factor. Studies on posttransfusion and dissociated factor VIII and in patients with von Willebrand's disease. J Clin Invest. 1977; 60:390-404. [PubMed: 17621]

3. Sadler JE, Mannucci PM, Berntorp E, et al. Impact, diagnosis and treatment of von Willebrand disease. Thromb Haemost. 2000; 84:160-174. [PubMed: 10959685]

4. Montgomery RR, Kroner PA. von Willebrand disease: a common pediatric disorder. Pediatr Ann. 2001; 30:534-540. [PubMed: 11554262]

5. Verweij CL, Diergaarde PJ, Hart M, Pannekoek H. Full-length von Willebrand factor (vWF) cDNA encodes a highly repetitive protein considerably larger than the mature vWF subunit [published erratum appears in EMBO J. 1986;5:3074]. EMBO J. 1986; 5:1839-1847. [PubMed: 3019665]

6. Wagner DD, Olmsted JB, Marder VJ. Immunolocalization of von Willebrand protein in WeibelPalade bodies of human endothelial cells. J Cell Biol. 1982; 95:355-360. [PubMed: 6754744]

7. Sporn LA, Chavin SI, Marder VJ, Wagner DD. Biosynthesis of von Willebrand protein by human megakaryocytes. J Clin Invest. 1985; 76:1102-1106. [PubMed: 2413071]

8. Jaffe EA, Hoyer LW, Nachman RL. Synthesis of antihemophilic factor antigen by cultured human endothelial cells. J Clin Invest. 1973; 52:2757-2764. [PubMed: 4583980]

9. Nachman R, Levine R, Jaffe EA. Synthesis of factor VIII antigen by cultured guinea pig megakaryocytes. J Clin Invest. 1977; 60:914-921. [PubMed: 70434]

10. Wagner DD. Cell biology of von Willebrand factor. Annu Rev Cell Biol. 1990; 6:217-246. [PubMed: 2275814]

11. Carew JA, Quinn SM, Stoddart JH, Lynch DC. O-linked carbohydrate of recombinant von Willebrand factor influences ristocetin-induced binding to platelet glycoprotein 1b. J Clin Invest. 1992; 90:2258-2267. [PubMed: 1469086]

12. Carew JA, Browning PJ, Lynch DC. Sulfation of von Willebrand factor. Blood. 1990; 76:25302539. [PubMed: 2265247] 
13. Vischer UM, Wagner DD. von Willebrand factor proteolytic processing and multimerization precede the formation of Weibel-Palade bodies. Blood. 1994; 83:3536-3544. [PubMed: 8204880]

14. Wagner DD. The Weibel-Palade body: the storage granule for von Willebrand factor and Pselectin. Thromb Haemost. 1993; 70:105-110. [PubMed: 7694385]

15. Tsai HM, Nagel RL, Hatcher VB, Sussman II. Multimeric composition of endothelial cell-derived von Willebrand factor. Blood. 1989; 73:2074-2076. [PubMed: 2786433]

16. Moake JL, Turner NA, Stathopoulos NA, Nolasco LH, Hellums JD. Involvement of large plasma von Willebrand factor (vWF) multimers and unusually large vWF forms derived from endothelial cells in shear stress-induced platelet aggregation. J Clin Invest. 1986; 78:1456-1461. [PubMed: 3491092]

17. Wagner DD, Saffaripour S, Bonfanti R, et al. Induction of specific storage organelles by von Willebrand factor propolypeptide. Cell. 1991; 64:403-413. [PubMed: 1988154]

18. Voorberg J, Fontijn R, van Mourik JA, Pannekoek H. Domains involved in multimer assembly of von Willebrand factor (vWF): multimerization is independent of dimerization. EMBO J. 1990; 9:797-803. [PubMed: 2311582]

19. Haberichter SL, Jozwiak MA, Rosenberg JB, Christopherson PA, Montgomery RR. The von Willebrand factor propeptide (VWFpp) traffics an unrelated protein to storage. Arterioscler Thromb Vasc Biol. 2002; 22:921-926. [PubMed: 12067899]

20. Haberichter SL, Jacobi P, Montgomery RR. Critical independent regions in the VWF propeptide and mature VWF that enable normal VWF storage. Blood. 2003; 101:1384-1391. [PubMed: 12393513]

21. Haberichter SL, Fahs SA, Montgomery RR. Von Willebrand factor storage and multimerization: 2 independent intracellular processes. Blood. 2000; 96:1808-1815. [PubMed: 10961880]

22. Hannah MJ, Williams R, Kaur J, Hewlett LJ, Cutler DF. Biogenesis of Weibel-Palade bodies. Semin Cell Dev Biol. 2002; 13:313-324. [PubMed: 12243731]

23. van Mourik JA, Romani DW, Voorberg J. Biogenesis and exocytosis of Weibel-Palade bodies. Histochem Cell Biol. 2002; 117:113-122. [PubMed: 11935287]

24. Sanders WE Jr, Reddick RL, Nichols TC, Brinkhous KM, Read MS. Thrombotic thrombocytopenia induced in dogs and pigs. The role of plasma and platelet vWF in animal models of thrombotic thrombocytopenic purpura. Arterioscler Thromb Vasc Biol. 1995; 15:793800. [PubMed: 7773736]

25. Denis C, Methia N, Frenette PS, et al. A mouse model of severe von Willebrand disease: defects in hemostasis and thrombosis. Proc Natl Acad Sci U S A. 1998; 95:9524-9529. [PubMed: 9689113]

26. Olsen EH, McCain AS, Merricks EP, et al. Comparative response of plasma VWF in dogs to upregulation of VWF mRNA by interleukin-11 versus Weibel-Palade body release by desmopressin (DDAVP). Blood. 2003; 102:436-441. [PubMed: 12649145]

27. Rosenberg JB, Haberichter SL, Jozwiak MA, et al. The role of the D1 domain of the von Willebrand factor propeptide in multimerization of VWF. Blood. 2002; 100:1699-1706. [PubMed: 12176890]

28. Nichols TC, Bellinger DA, Reddick RL, et al. The roles of von Willebrand factor and factor VIII in arterial thrombosis: studies in canine von Willebrand disease and hemophilia A. Blood. 1993; 81:2644-2651. [PubMed: 8490173]

29. Hop C, Guilliatt A, Daly M, et al. Assembly of multimeric von Willebrand factor directs sorting of P-selectin. Arterioscler Thromb Vasc Biol. 2000; 20:1763-1768. [PubMed: 10894814]

30. Michaux G, Hewlett LJ, Messenger SL, et al. Analysis of intracellular storage and regulated secretion of 3 von Willebrand disease-causing variants of von Willebrand factor. Blood. 2003; 102:2452-2458. [PubMed: 12791651]

31. Blagoveshchenskaya AD, Hannah MJ, Allen S, Cutler DF. Selective and signal-dependent recruitment of membrane proteins to secretory granules formed by heterologously expressed von Willebrand factor. Mol Biol Cell. 2002; 13:1582-1593. [PubMed: 12006654]

32. Bonfanti R, Furie BC, Furie B, Wagner DD. PADGEM (GMP140) is a component of WeibelPalade bodies of human endothelial cells. Blood. 1989; 73:1109-1112. [PubMed: 2467701] 
33. McEver RP, Beckstead JH, Moore KL, Marshall-Carlson L, Bainton DF. GMP-140, a platelet alpha-granule membrane protein, is also synthesized by vascular endothelial cells and is localized in Weibel-Palade bodies. J Clin Invest. 1989; 84:92-99. [PubMed: 2472431]

34. Blagoveshchenskaya AD, Norcott JP, Cutler DF. Lysosomal targeting of P-selectin is mediated by a novel sequence within its cytoplasmic tail. J Biol Chem. 1998; 273:2729-2737. [PubMed: 9446579]

35. Venta PJ, Li J, Yuzbasiyan-Gurkan V, Brewer GJ, Schall WD. Mutation causing von Willebrand's disease in Scottish Terriers. J Vet Intern Med. 2000; 14:10-19. [PubMed: 10668811]

36. Denis CV, Andre P, Saffaripour S, Wagner DD. Defect in regulated secretion of P-selectin affects leukocyte recruitment in von Willebrand factor-deficient mice. Proc Natl Acad Sci U S A. 2001; 98:4072-4077. [PubMed: 11274431]

37. Blagoveshchenskaya AD, Hewitt EW, Cutler DF. A complex web of signal-dependent trafficking underlies the triorganellar distribution of P-selectin in neuroendocrine PC12 cells. J Cell Biol. 1999; 145:1419-1433. [PubMed: 10385522]

38. Green SA, Setiadi H, McEver RP, Kelly RB. The cytoplasmic domain of P-selectin contains a sorting determinant that mediates rapid degradation in lysosomes. J Cell Biol. 1994; 124:435-448. [PubMed: 7508941]

39. Voorberg J, Fontijn R, Calafat J, et al. Biogenesis of von Willebrand factor-containing organelles in heterologous transfected CV-1 cells. EMBO J. 1993; 12:749-758. [PubMed: 8440262]

40. Arvan P, Castle D. Sorting and storage during secretory granule biogenesis: looking backward and looking forward. Biochem J. 1998; 332(Pt 3):593-610. [PubMed: 9620860]

41. Tooze SA. Biogenesis of secretory granules in the trans-Golgi network of neuroendocrine and endocrine cells. Biochim Biophys Acta. 1998; 1404:231-244. [PubMed: 9714820]

42. Padilla A, Moake JL, Bernardo A, et al. P-selectin anchors newly released ultra-large von Willebrand factor multimers to the endothelial cell surface. Blood. 2004; 103:2150-2156. [PubMed: 14630802]

43. Huber D, Cramer EM, Kaufmann JE, et al. Tissue-type plasminogen activator (t-PA) is stored in Weibel-Palade bodies in human endothelial cells both in vitro and in vivo. Blood. 2002; 99:36373645. [PubMed: 11986218]

44. Romani, dW; de Leeuw, HP.; Rondaij, MG., et al. Von Willebrand factor targets IL-8 to WeibelPalade bodies in an endothelial cell line. Exp Cell Res. 2003; 286:67-74. [PubMed: 12729795]

45. Wolff B, Burns AR, Middleton J, Rot A. Endothelial cell "memory" of inflammatory stimulation: human venular endothelial cells store interleukin 8 in Weibel-Palade bodies. J Exp Med. 1998; 188:1757-1762. [PubMed: 9802987]

46. Do H, Healey JF, Waller EK, Lollar P. Expression of factor VIII by murine liver sinusoidal endothelial cells. J Biol Chem. 1999; 274:19587-19592. [PubMed: 10391893]

47. Rosenberg JB, Greengard JS, Montgomery RR. Genetic induction of a releasable pool of factor VIII in human endothelial cells. Arterioscler Thromb Vasc Biol. 2000; 20:2689-2695. [PubMed: 11116073] 


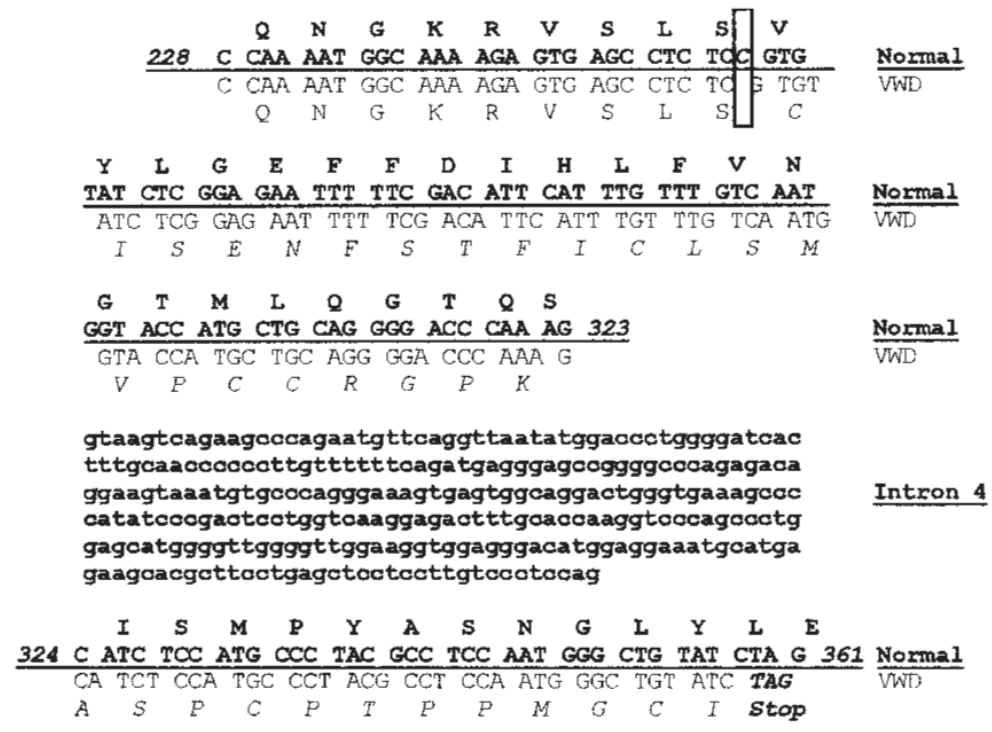

Figure 1. A homozygous frameshift mutation results in premature termination of VWF protein synthesis

Genomic DNA obtained from normal and VWD dogs was sequenced, and the 2 sequences were compared, including sequence from exon 4 , intron 4 , and exon 5 . The protein sequence is shown above the corresponding nucleic acid sequence with normal canine sequence shown in bold font above the VWD canine sequence. A single nucleotide deletion was found in the VWD canine sequence at base pair 255 as highlighted in the box, resulting in a frameshift. The altered VWD canine protein sequence is shown in italics. The altered VWD canine nucleotide sequence results in a stop codon at base pair 359 in exon 5. 

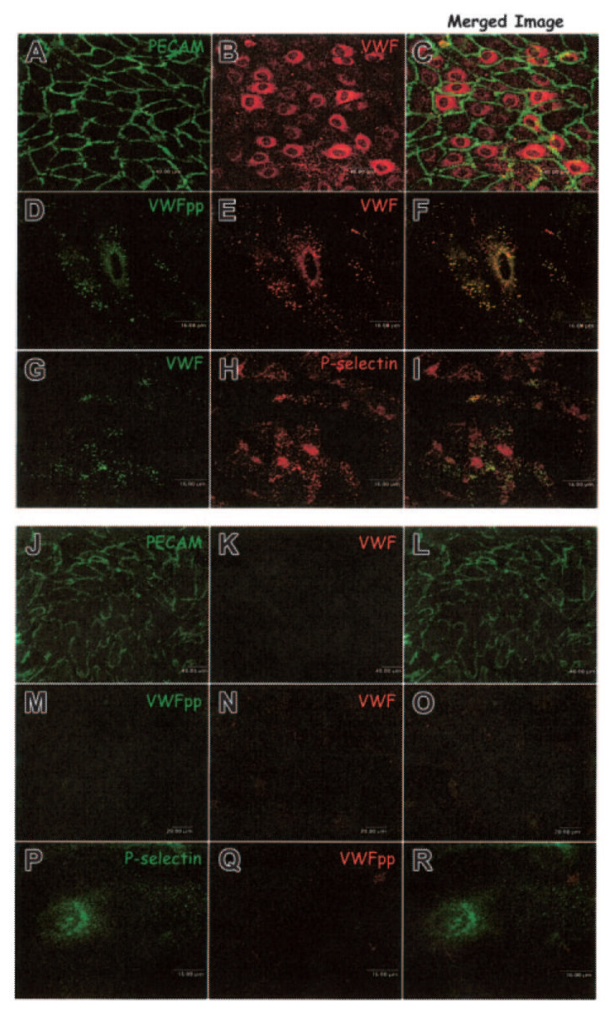

Figure 2. Aortic endothelial cells obtained from a normal dog maintain a normal Weibel-Palade body distribution of VWFpp, VWF, and P-selectin while canine VWD aortic endothelial cells neither express VWF nor contain Weibel-Palade bodies

(A-I) Endothelial cells were harvested from the aortas of normal dogs, grown on gelatincoated slides, fixed, permeabilized, immunostained, and examined by confocal microscopy as described in "Materials and methods." Cells immunostained with a mouse monoclonal anti-PECAM (A) and a polyclonal anti-VWF (B) demonstrated a homogeneous population of endothelial cells. Staining with a mixture of several mouse monoclonal anti-VWFpp (D) and a polyclonal anti-VWF (E) demonstrated the presence of both proteins in granules where they colocalized ( $\mathrm{F}$, colocalization is indicated by yellow pixels). Immunostaining of the normal endothelial cells with the mouse monoclonal anti-VWF, AVW-1 (G), and rabbit anti-P-selectin $(\mathrm{H})$ revealed that VWF was colocalized with P-selectin in granules (I). The aortic endothelial cells from normal dogs maintain characteristic Weibel-Palade bodies. (JR) Endothelial cells were harvested from the aortas of type 3 VWD dogs, cultured, immunostained, and examined by confocal microscopy. Cells were dual-labeled with mouse monoclonal anti-PECAM (J) and polyclonal anti-VWF (K). The homogeneous population of endothelial cells was VWF-negative. Neither VWF nor VWFpp could be detected in these cells by immunostaining with a mix of several mouse monoclonal anti-VWFpp antibodies (M) and polyclonal anti-VWF (N). Cells were also labeled with a rabbit anti-P-selectin antibody (P) in addition to the canine-specific monoclonal VWFpp antibody (Q). Staining for P-selectin was faint and diffuse with many small granules (P). No apparent WeibelPalade bodies were detected in the canine VWD aortic endothelial cells. 


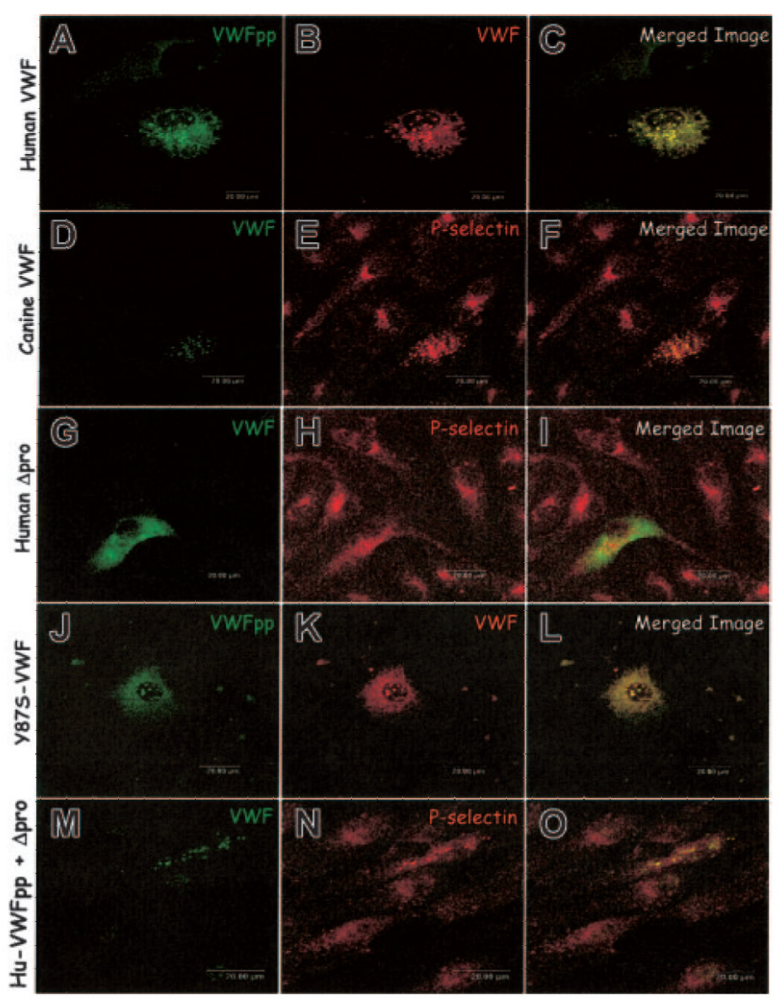

Figure 3. Expression of human or canine VWF proteins in canine VWD aortic endothelial cells (A-F) Wild-type VWF. Canine VWD aortic endothelial cells were transiently transfected with either human VWF (A-C) or canine VWF (D-F) by nucleofection. Transfected cells were fixed, immunolabeled, and examined by confocal microscopy as described in "Materials and methods." Cells were immunolabeled with a mix of several mouse monoclonal anti-VWFpp antibodies (A), a rabbit anti-VWF (B), AVW-1 mouse monoclonal anti-VWF (D), or rabbit anti-P-selectin (E). Immunostaining of cells expressing human VWF demonstrated a granular distribution of VWFpp and VWF (A-B). Merging the 2 images revealed colocalization of the proteins $(\mathrm{C})$. Dual staining for VWF and P-selectin revealed that the canine VWF (D-F) colocalized with P-selectin in granules. Expression of canine VWF reestablishes the Weibel-Palade body distribution of endogenous P-selectin. (G-L) Dimeric VWF. Cells were transiently transfected with either a dimeric, propeptidedeleted VWF ( $\Delta$ pro) or dimeric, mutant Y87S-VWF. Cells were immunostained using the monoclonal anti-VWF, AVW-1 (G), rabbit anti-P-selectin (H), a mix of several mouse monoclonal anti-VWFpp antibodies $(\mathrm{J})$ or polyclonal anti-VWF $(\mathrm{K})$. Cells expressing human $\Delta$ pro (G-I) showed diffuse VWF staining with no apparent VWF-containing granules (G). P-selectin distribution was unchanged $(\mathrm{H})$, and the 2 proteins did not appear to colocalize (I). A dimeric, Y87S-VWF mutant was also transiently expressed in the canine VWD aortic endothelial cells (J-L). The mutant Y87S VWFpp and VWF showed a granular staining pattern in addition to diffuse staining and were found to colocalize (J-L). VWF multimerization is not necessary for Weibel-Palade body formation. (M-O) Coexpression of VWFpp and $\Delta$ pro. Cells were transiently transfected with $\Delta$ pro and VWFpp in trans as 2 separate constructs. Cells were immunostained using AVW-1 monoclonal anti-VWF (M) and rabbit anti-P-selectin $(\mathrm{N})$. In cells expressing VWF, P-selectin was found to colocalize with VWF in granules (O). Coexpression of VWFpp and $\Delta$ pro generates Weibel-Palade bodies in canine VWD aortic endothelial cells. 
A
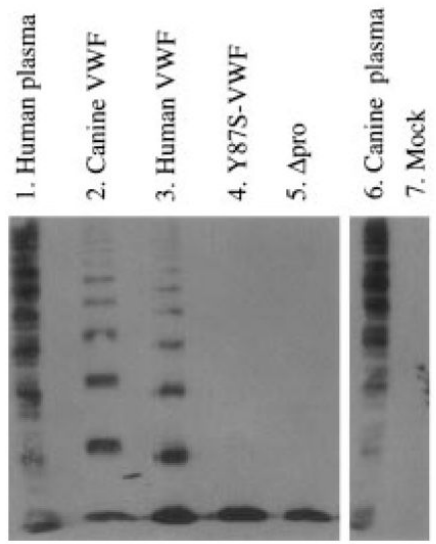

B

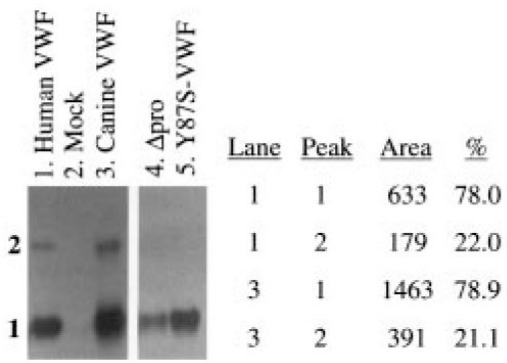

Figure 4. Multimeric structure of expressed canine and human VWF proteins

(A) The multimeric structure of expressed VWF constructs was analyzed nonreduced on a $3 \%$ agarose-SDS gel. All samples were run on the same gel, and interfering (nonrelevant) lanes have been removed for clarity. No VWF was detected in the mock-transfected control (lane 7). Wild-type canine and human full-length VWF (lanes 2, 3) and normal human and canine plasmas (lanes 1 and 6) were normally multimerized. Neither Y87S-VWF (lane 4) nor $\Delta$ pro (propeptide-deleted VWF, lane 5) formed high molecular weight multimers. (B) The structure of lower molecular weight VWF subunits was analyzed on a 3\% MetaPhoragarose submarine gel. No VWF was detected in the mock-transfected control (lane 2). Y87S-VWF and $\Delta$ pro formed only a dimeric VWF species (lanes 4,5 ). The density of the dimer and tetramer bands observed in human and canine VWF (lanes 1, 3) were calculated after scanning the immunoblot (peak 1 = dimer). 

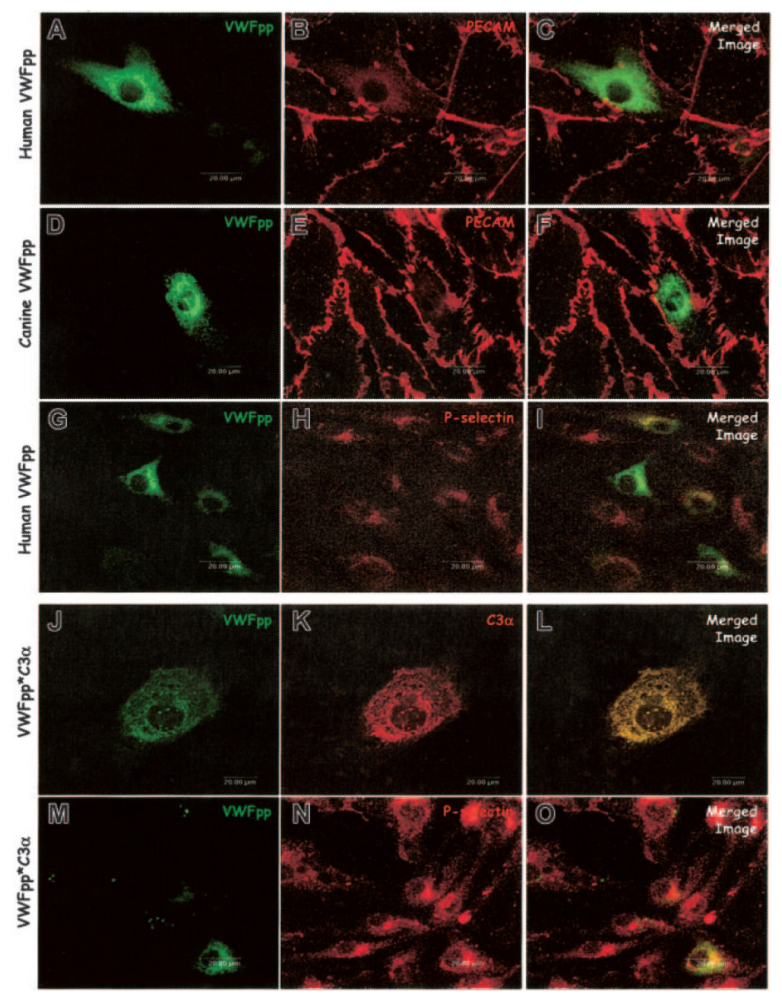

Figure 5. VWFpp alone does not induce Weibel-Palade body formation

The VWFpp and the fusion protein VWFpp*C3a were independently expressed in canine VWD aortic endothelial cells. Transfected cells were processed and examined as described in "Materials and methods." Cells were immunostained with a rabbit anti-VWFpp (A, D), a mix of several monoclonal anti-VWFpp antibodies (G, J, M), mouse anti-PECAM (B, E), rabbit-anti-P-selectin $(\mathrm{H}, \mathrm{N})$, or polyclonal anti-C3 antibody $(\mathrm{K})$. In cells expressing either human VWFpp (A-C) or canine VWFpp (D-F), the distribution of VWFpp was diffuse, although small VWFpp-containing granules were occasionally observed (D). Cells expressing human VWFpp were dual stained for VWFpp (G) and P-selectin (H): P-selectin distribution was unchanged compared with untransfected VWD endothelial cells and very little colocalization of VWFpp and P-selectin was observed (I). Cells expressing the VWFpp*C3a fusion protein showed both diffuse staining as well as granular storage of both VWFpp (J) and C3a (K). Dual-staining for VWFpp (M) and P-selectin (N) revealed very little colocalization of the VWFpp*C3a protein with P-selectin (O). While the VWFpp*C3a protein may induce granule formation in the canine VWD aortic endothelial cells, P-selectin is not recruited to the newly formed granule. 

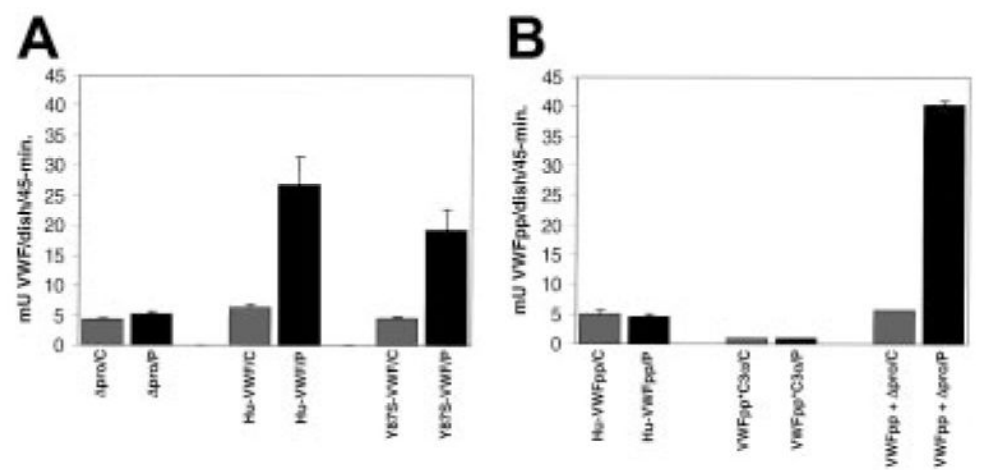

Figure 6. Agonist-induced release of expressed VWF proteins from canine VWD aortic endothelial cells

Canine VWD aortic endothelial cells were transiently transfected with $\Delta$ pro, wild-type VWF, Y87S-VWF, VWFpp, VWFpp*C3a, or VWFpp $+\Delta$ pro (trans). The culture media were harvested from transfected cells, the cells washed with HBSS, then cells were incubated with either $2 \mu \mathrm{M}$ PMA or OptiMEM (control) for 45 minutes. The incubation media were collected, and the amount of VWF or VWFpp was quantitated by ELISA. (A) The concentration of released $\Delta$ pro, wild-type VWF, and Y87S-VWF was determined by VWF ELISA. (Legend: $/ \mathrm{C}=$ control and $/ \mathrm{P}=\mathrm{PMA}$ treated) (B) The concentration of released VWFpp, VWFpp*C3a, and VWFpp $+\Delta$ pro (trans) were determined by VWFpp ELISA. Wild-type VWF, Y87S-VWF, and VWFpp + $\Delta$ pro (trans) were released in response to PMA, while $\Delta$ pro, VWFpp, and VWFpp*C3a did not show a significant increase in protein release. 